\title{
Healthy Behaviors, Risk Factor Control and Awareness of Chronic Kidney Disease
}

\author{
Delphine S. Tuot $^{\mathrm{a}}$ Laura C. Plantinga $^{\mathrm{f}}$ Suzanne E. Judd ${ }^{\mathrm{b}}$ Paul Muntner $^{\mathrm{b}, \mathrm{c}}$ \\ Chi-yuan Hsu $^{a}$ David G. Warnock ${ }^{d}$ Orlando M. Gutiérrez ${ }^{d}$ Monika Safford ${ }^{d}$ \\ Neil R. Powe ${ }^{a}$ William M. McClellan ${ }^{e}$ for the REGARDS Investigators \\ ${ }^{a}$ Department of Medicine, University of California at San Francisco, San Francisco, Calif., ${ }^{b}$ Departments of \\ Biostatistics and Epidemiology, 'School of Public Health, University of Alabama at Birmingham and \\ ${ }^{d}$ Department of Medicine, University of Alabama at Birmingham, Birmingham, Ala., and Departments of \\ ${ }^{\mathrm{e}}$ Medicine and ${ }^{\mathrm{f}}$ Epidemiology, Emory University, Atlanta, Ga., USA
}

\section{Key Words}

Chronic kidney disease - Awareness · Self-management . Behaviors

\begin{abstract}
Background/Aims: The association between chronic kidney disease (CKD) awareness and healthy behaviors is unknown. We examined whether CKD self-recognition is associated with healthy behaviors and achieving risk-reduction targets known to decrease risk of cardiovascular morbidity and CKD progression. Methods: CKD awareness, defined as a 'yes' response to 'Has a doctor or other health professional ever told you that you had kidney disease?', was examined among adults with CKD (eGFR $<60 \mathrm{ml} / \mathrm{min} / 1.73 \mathrm{~m}^{2}$ ) who participated in the REasons for Geographic And Racial Differences in Stroke (REGARDS) study. Odds of participation in healthy behaviors (tobacco avoidance, avoidance of regular nonsteroidal anti-inflammatory drug use, and physical activity) and achievement of risk-reduction targets (angiotensin-converting enzyme inhibitor/angiotensin receptor blocker use, systolic blood pressure control and glycemic control among those with diabetes) among those aware versus unaware of their CKD were determined by logistic regression, controlling for sociodemographics, access to care and comorbid
\end{abstract}

conditions. Systolic blood pressure control was defined as $<130 \mathrm{~mm} \mathrm{Hg}$ (primary definition) or $<140 \mathrm{~mm} \mathrm{Hg}$ (secondary definition). Results: Of 2,615 participants, only $6 \%(n=166)$ were aware of having CKD. Those who were aware had $82 \%$ higher odds of tobacco avoidance compared to those unaware (adjusted OR $=1.82,95 \% \mathrm{Cl} 1.02-3.24$ ). CKD awareness was not associated with other healthy behaviors or achievement of risk-reduction targets. Conclusions: Awareness of CKD was only associated with participation in one healthy behavior and was not associated with achievement of risk-reduction targets. To encourage adoption of healthy behaviors, a better understanding of barriers to participation in CKD-healthy behaviors is needed.

Copyright $\odot 2013$ S. Karger AG, Basel

\section{Introduction}

Chronic kidney disease (CKD) is common, affecting at least 20 million Americans or up to $11.5 \%$ of the United States population [1]. CKD is associated with excess mortality, morbidity and diminished quality of life $[2,3]$. It is thought that patient adoption of healthy risk-reduction behaviors, such as tobacco avoidance [4] and participating in physical activity [5] may improve health

\section{KARGER}

E-Mail karger@karger.com

www.karger.com/ajn
(C) 2013 S. Karger AG, Basel

0250-8095/13/0372-0135\$38.00/0
Delphine S. Tuot, MDCM, MAS

Division of Nephrology, San Francisco General Hospital

1001 Potrero Avenue, Building 100, Room 342

San Francisco, CA 94110 (USA)

E-Mail Delphine.tuot@ucsf.edu 
outcomes, particularly by decreasing cardiovascular risk and slowing CKD progression. Recent efforts have focused on encouraging lifestyle changes among patients with severe kidney disease. For example, Medicare currently reimburses clinicians who provide predialysis education [6] focused on such topics as: diet/nutrition, exercise, and managing comorbid conditions. Such programs were developed because there is evidence that predialysis education leads to delayed initiation of dialysis [7], improved self-care [8], and decreased mortality [9] among patients with end-stage renal disease (ESRD) receiving dialysis.

Previous studies have reported that self-recognition of moderate CKD is very low [10], as is actual patient knowledge about kidney disease and its complications [11]. Despite this, few self-management support or educational programs that focus on increasing awareness and selfcare have been implemented among patients with early or moderate CKD [12]. The lack of evidence linking CKD awareness and healthy behaviors among individuals with moderate CKD (unlike among individuals with ESRD) may be one barrier to the development of such programs.

The aim of this study was to examine whether a person's awareness of his/her CKD is associated with selfreported healthy behaviors and risk-reduction targets. We hypothesized that CKD awareness among patients with CKD not requiring renal replacement therapy would be associated with participation in behaviors and achievement of risk-reduction targets thought to decrease risk of adverse health outcomes. Behaviors included tobacco avoidance [4], avoidance of regular use of nonsteroidal anti-inflammatory drugs (NSAIDs) [13], and increased physical activity [5]; risk-reduction targets included systolic blood pressure (SBP) control [14], use of angiotensin-converting enzyme inhibitors (ACEI) or angiotensin receptor blockers (ARB) [14], and glycemic control among patients with $\mathrm{CKD}$ and diabetes.

\section{Methods}

\section{Study Design}

We used data previously collected from the NIH-funded REasons for Geographic And Racial Differences in Stroke (REGARDS) study cohort. The purpose of REGARDS was to identify factors that contribute to the excess stroke mortality among black individuals and residents of the Southeastern United States. Methods for recruitment in REGARDS have been described elsewhere [15]. By design, the REGARDS study cohort was restricted to adults $\geq 45$ years of age. Black individuals ( $42 \%$ of the final cohort), as well as individuals who live in the 'stroke buckle' (coastal North Carolina, South Carolina, and Georgia) or the 'stroke belt' (remainder of North Carolina, South Carolina and Georgia as well as Tennessee, Mississippi, Alabama and Arkansas), were oversampled. Residents of the stroke belt/buckle comprised $56 \%$ of the final cohort; the other $44 \%$ of the study cohort resided in the remaining continental US.

Overall, 30,239 participants were recruited between January 2003 and October 2007. A question about awareness of CKD was added to the baseline REGARDS study questionnaire in May 2004 and was available for 21,658 participants. We further restricted our analysis to REGARDS study participants with an estimated glomerular filtration rate (eGFR) $15-59 \mathrm{ml} / \mathrm{min} / 1.73 \mathrm{~m}^{2}(\mathrm{n}=3,020$, $14 \%$ of those asked about awareness of CKD). We excluded participants who had missing data about CKD awareness, laboratory and health behavior data $(\mathrm{n}=405,13 \%$ of study participants with eGFR $15-59 \mathrm{ml} / \mathrm{min} / 1.73 \mathrm{~m}^{2}$ ), resulting in a final study population of 2,615 participants. The REGARDS study protocol was approved by Institutional Review Boards governing research in human subjects at participating centers. All participants provided informed consent.

\section{Data Collection}

Trained interviewers conducted computer-assisted telephone interviews to obtain participants' sociodemographic data (age, sex, race, social support, income, education), information about access to care (insurance status, last visit with a physician), and self-reported diagnoses of comorbid conditions, including CKD, diabetes, hypertension, coronary heart disease (history of coronary artery bypass, angioplasty or stent placement) and stroke.

Health professionals conducted in-home baseline study visits that included a physical examination, collection of either fasting (>90\%) or nonfasting blood samples, random (non-first morning) urine samples, and an inventory of current medications. BP was measured two times during the exam following a standardized protocol; the mean of the measurements was used. An ECG was performed. Serum creatinine assays were calibrated with an isotope dilution mass spectroscopic standard. Urinary albumin was measured using the BN ProSpec Nephelometer from Dade Behring (Marburg, Germany). Urinary creatinine was measured with a rate-blanked Jaffé procedure, using the Modular-P analyzer (Roche/Hitachi, Indianapolis, Ind., USA) and calibrated to an isotope-dilution mass spectroscopy standard; urine albumin:creatinine ratio results were expressed in $\mathrm{mg} / \mathrm{g}$.

\section{Definitions}

Hypertension was defined by self-reported use of antihypertensive medications or a SBP/DBP $\geq 140 / 90 \mathrm{~mm} \mathrm{Hg}$. Diabetes was considered present if self-reported or if the participant reported use of oral-hypoglycemic medications or insulin, or had a fasting serum glucose $\geq 126 \mathrm{mg} / \mathrm{dl}$ or, for nonfasting participants, a serum glucose $\geq 200 \mathrm{mg} / \mathrm{dl}$. Coronary heart disease was defined by selfreport or if there was evidence of a previous myocardial infarction on ECG. Stroke was self-reported. The CKD-EPI equation [1] was used to estimate GFR; CKD was defined by the Kidney Disease Outcomes Quality Initiative staging guidelines [16].

\section{Predictor}

The primary predictor was individual awareness of CKD. Participants who responded 'yes' to 'Has a doctor or other health professional ever told you that you had kidney disease?' were defined as being aware of their CKD. 


\section{Outcomes}

Self-reported health behaviors thought to influence risk of adverse health outcomes were obtained during the computer-assisted telephone interviews. Current tobacco avoidance was defined by a 'no' answer to 'Have you smoked at least 100 cigarettes in your lifetime' or a 'yes' answer to that question, followed by a 'no' response to 'Do you smoke cigarettes now, even occasionally?' Chronic NSAID use was defined by a 'yes' reply to 'Are you currently taking nonsteroidal anti-inflammatory agents on a regular basis, that is at least two times each week?' after an exhaustive list of NSAID examples were provided. Frequency and chronicity of NSAID use was self-reported, as data obtained from the medication inventory might misclassify sporadic NSAID use. Physical activity was determined by the question 'How many times per week do you engage in intense physical activity, enough to work up a sweat?' Answers were categorized into 3 groups: none, occasional (1-3 times per week) and frequent (4+ times per week).

Risk-reduction targets were ascertained during the in-home study visit. Current ACEI/ARB use (yes/no) was determined during the medication inventory. SBP control was defined in two ways given the current debate on target SBP control among patients with CKD [16]: mean SBP $<130 \mathrm{~mm} \mathrm{Hg}$ (primary definition) or $<140 \mathrm{~mm} \mathrm{Hg}$ (secondary definition).

Among individuals with CKD and self-reported hypertension, ACEI/ARB use and SBP control were examined. Among individuals with $\mathrm{CKD}$ and self-reported diabetes, $\mathrm{ACEI} / \mathrm{ARB}$ use and glycemic control were examined. Because we did not have access to glycosylated hemoglobin data, glycemic control was defined by a preprandial blood glucose $<130 \mathrm{mg} / \mathrm{dl}$, per American Diabetes Association recommendations [17].

\section{Statistical Methods}

Differences among participants with complete data and missing data were calculated and compared using $\chi^{2}$ tests. Among participants with complete data, differences between those aware and unaware of CKD were calculated using $\chi^{2}$ tests. Multivariate logistic regression models were used to examine the independent association between CKD awareness and the aforementioned outcome variables. The interaction terms awareness $\times$ income and awareness $\times$ education were initially included in models to explore whether socioeconomic status modified the association between CKD awareness and healthy behaviors/risk-reduction targets. These terms were nonsignificant ( $\mathrm{p}>0.2$ for all unadjusted models) and thus dropped from the final models. Analyses were adjusted for covariates in an incremental fashion, resulting in 3 models, adjusting for the following: (1) sociodemographics only (age, race, gender, marital status, income, education, region); (2) sociodemographics and comorbid conditions (high blood pressure, diabetes, stroke and coronary heart disease), and (3) sociodemographics, comorbid conditions and CKD severity (eGFR and urine albumin:creatinine ratio, both modeled as categorical variables). Similar incremental analyses were performed in two subgroups defined a priori: participants with CKD and hypertension and participants with CKD and diabetes. Analyses were conducted using SAS v. 9.3 (Cary, N.C., USA).

A sensitivity analysis was performed using the Modified Diet in Renal Disease (MDRD) study equation to calculate the eGFR [18], as this equation was commonly used in practice to determine CKD severity during the time of REGARDS participant recruitment.

\section{Results}

\section{Participants}

Our study population consisted of 2,615 individuals with CKD, among whom $6.3 \%(n=166)$ were aware of their CKD. These individuals differed from study subjects with missing data ( $\mathrm{n}=405)$, in that those with missing data tended to be younger $(\mathrm{p}=0.02)$, of Black race $(\mathrm{p}=$ $0.001)$, had more proteinuria $(\mathrm{p}=0.002)$ and lower eGFR $(\mathrm{p}=0.03)$. The two populations did not differ in regards to CKD awareness ( $\mathrm{p} \geq 0.05$ ).

Roughly one-half of the study sample was older than 70 years, with fairly even distribution with respect to gender and race (white and black) (table 1). The majority of the study sample had access to health care, with $97 \%$ reporting having medical insurance and $79 \%$ having seen a physician within the past year. Nearly $80 \%$ of the study sample had hypertension and 33\% had diabetes.

Individuals aware of their CKD with complete data were younger and more likely to be male than their counterparts unaware of CKD (table 1). Mean age among those aware and unaware of their CKD was 67.7 years (SD 9.3) and 72.6 years (SD 8.7), respectively. There was no statistically significant association between CKD awareness and household income $(\mathrm{p}=0.15)$ or race $(\mathrm{p}=0.09)$. Modest, nonsignificant higher CKD awareness was present among individuals with comorbid conditions, particularly among those with diabetes (adjusted odds ratio [AOR] for awareness 1.43, 95\% CI 0.96-2.12) and stroke $(\mathrm{AOR}=1.45,95 \%$ CI 0.89-2.38). Mean (SD) glucose values were not statistically different across both groups of study participants (unaware, $138.7 \mathrm{mg} / \mathrm{dl}$ (61.7); aware, $123.9 \mathrm{mg} / \mathrm{dl}$ (71.1); $\mathrm{p}=0.81)$; however, a significantly greater percentage of those aware of their $\mathrm{CKD}$ required insulin to manage their diabetes $(61.4 \%)$ versus those who were unaware of their CKD (38.1\%) ( $p<0.001)$. Awareness was higher among individuals with greater albuminuria and lower levels of eGFR ( $p<0.001$ for both).

\section{Association of CKD Awareness and Healthy \\ Behaviors/Risk-Reduction Targets}

The overall prevalence of healthy behaviors ranged from $24.0 \%$ (frequent physical activity) to $89.4 \%$ (tobacco avoidance), with nonsignificant differences among those aware versus those unaware of their CKD. Achievement of risk-reduction targets ranged from 49.3\% (SBP <130 $\mathrm{mm} \mathrm{Hg}$ ) to $72.5 \%$ (SBP $<140 \mathrm{~mm} \mathrm{Hg}$ ), again with nonsignificant differences between those aware and unaware of their CKD (fig. 1). 


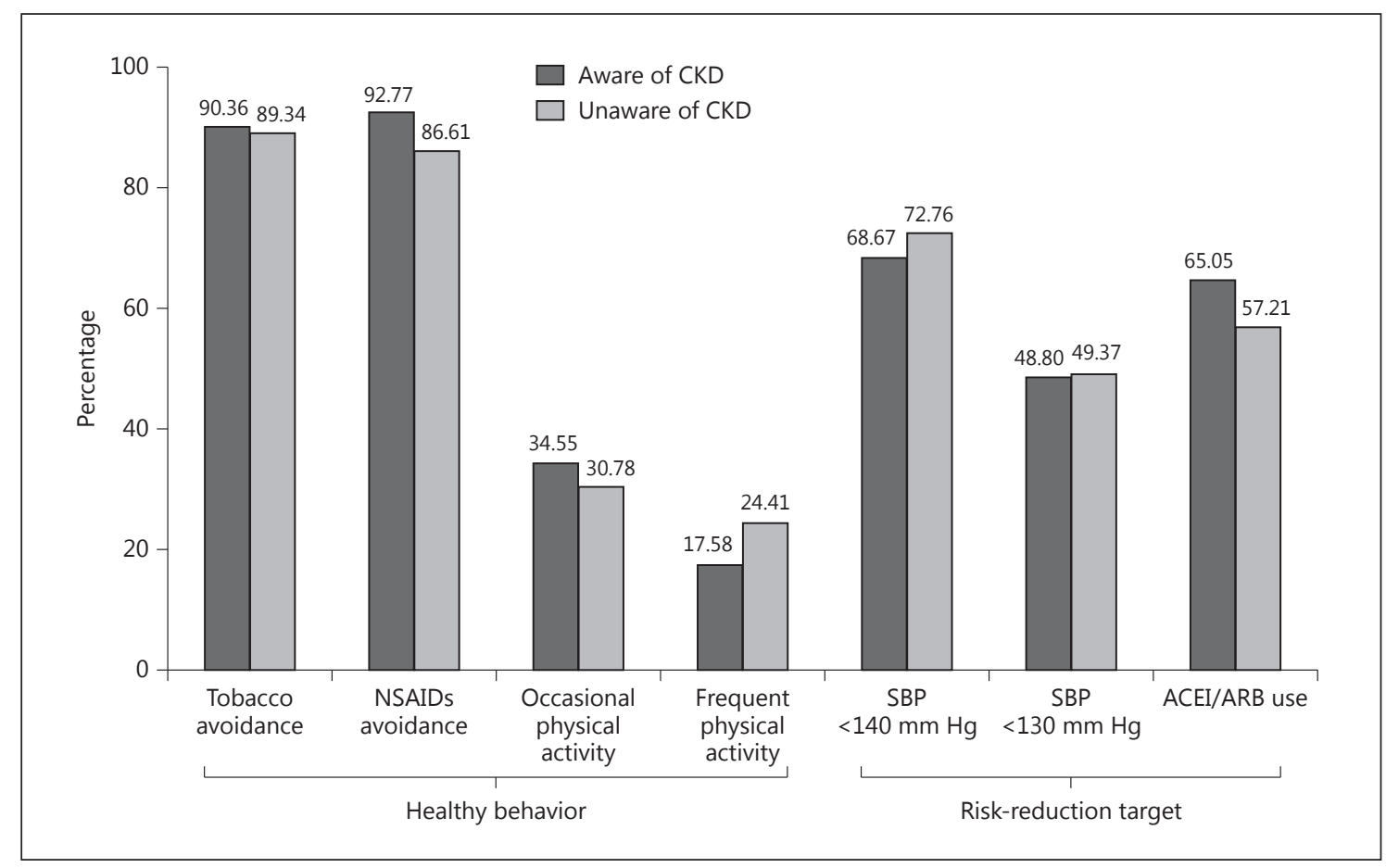

Fig. 1. Prevalence of healthy behaviors and achievement of risk-reduction targets among REGARDS participants with CKD, by awareness ( $\mathrm{p}>0.05$ for all unadjusted comparisons).

CKD awareness was not associated with participation in healthy behaviors. Adjusted for sociodemographic variables, $\mathrm{CKD}$ awareness was not associated with tobacco avoidance, NSAID avoidance or increased physical activity. After adjustment for comorbid conditions and $\mathrm{CKD}$ severity, those who were aware of their $\mathrm{CKD}$ were more likely to report avoidance of tobacco $(\mathrm{AOR}=1.82$, 1.02-3.24). However, odds of NSAID avoidance and participation in increased physical activity were not different among those aware versus unaware of their CKD. Also, CKD awareness was not associated with higher use of ACEI/ARB or achievement of SBP control (table 2).

Similar results were found when examining the association between CKD awareness and risk-reduction targets in two subpopulations (table 3). Among participants with CKD and hypertension $(\mathrm{n}=2,102)$, CKD awareness was not associated with SBP control or ACEI/ARB use. Among study participants with CKD and diabetes $(\mathrm{n}=$ 933), neither glycemic control (AOR $=1.05,0.65-1.69$ ) nor ACEI/ARB use $(A O R=0.91,0.55-1.52)$ differed among individuals aware versus unaware of their CKD, after adjusting for sociodemographic characteristics, comorbid conditions, and CKD severity.

\section{Sensitivity Analysis}

Using the MDRD equation, 2,656 participants with complete data had eGFR $15-59 \mathrm{ml} / \mathrm{min} / 1.73 \mathrm{~m}^{2}$, of which 172 individuals (6.5\%) demonstrated CKD awareness. The overall prevalence of healthy behaviors differed slightly when using the MDRD equation to determine eGFR. Prevalence of moderate and frequent physical activity were 31 and 24\%, respectively. Unadjusted prevalence of NSAID avoidance was $86 \%$, with a statistically significant difference among those aware versus unaware of their CKD ( $\mathrm{p}=0.007)$. Prevalence of tobacco avoidance was $94 \%$. Achievement of risk-reduction targets ranged from $52 \%$ (SBP $<130 \mathrm{~mm} \mathrm{Hg}$ ), to $57 \%$ (use of ACEI/ARB) to $74 \%$ (SBP $<140 \mathrm{~mm} \mathrm{Hg}$ ), with significant differences between those aware and unaware of their CKD with respect to $\mathrm{SBP}<140 \mathrm{~mm} \mathrm{Hg}(\mathrm{p}=0.05)$ and ACEI/ARB use $(\mathrm{p}=0.03)$.

However, after adjusting for sociodemographic variables, comorbid conditions and CKD severity, CKD awareness was not associated with participation in healthy behaviors or achievement of risk-reduction targets, when using the MDRD study equation to calculate eGFR, with the exception of tobacco avoidance (online suppl. tables S1 and S2; see www.karger.com/doi/10.1159/000346712). 
Table 1. Characteristics of REGARDS study participants by CKD awareness

\begin{tabular}{|c|c|c|c|}
\hline & $\begin{array}{l}\text { Not aware of CKD } \\
(\mathrm{n}=2,449) \mathrm{n}(\%)\end{array}$ & $\begin{array}{l}\text { Aware of CKD } \\
(n=166) n(\%)\end{array}$ & $\begin{array}{l}\mathrm{OR}^{*}(95 \% \mathrm{CI}) \text { for } \\
\text { awareness }\end{array}$ \\
\hline \multicolumn{4}{|l|}{ Age } \\
\hline $45-59$ years & $164(6.7)$ & $31(18.6)$ & ref. \\
\hline $60-69$ years & $649(26.5)$ & $64(38.6)$ & $0.43(0.25,0.74)$ \\
\hline $70-79$ years & $1,046(42.7)$ & $51(30.7)$ & $0.23(0.13,0.42)$ \\
\hline $80+$ years & $590(24.1)$ & $20(12.1)$ & $0.17(0.08 .0 .33)$ \\
\hline \multicolumn{4}{|l|}{ Gender } \\
\hline Female & $1,353(55.3)$ & $70(42.2)$ & ref. \\
\hline Male & $1,096(44.8)$ & $96(57.8)$ & $1.67(1.10,2.53)$ \\
\hline \multicolumn{4}{|l|}{ Race } \\
\hline White & $1,461(59.7)$ & $88(53.0)$ & ref \\
\hline Black & $988(40.3)$ & $78(47.0)$ & $0.78(0.51,1.17)$ \\
\hline \multicolumn{4}{|l|}{ Marital status } \\
\hline Married & $1,218(49.7)$ & $70(42.2)$ & ref. \\
\hline Not married & $1,231(50.3)$ & $96(57.8)$ & $1.00(0.66,1.52)$ \\
\hline Education $<$ high school & $454(18.5)$ & $37(22.3)$ & $1.23(0.76,1.98)$ \\
\hline Income $<$ USD 20,000 per year & $574(23.4)$ & $47(28.3)$ & $1.02(0.65,1.60)$ \\
\hline \multicolumn{4}{|l|}{ Insurance status } \\
\hline No & $71(2.9)$ & $8(4.8)$ & ref. \\
\hline Yes & $2,376(97.1)$ & $158(95.2)$ & $1.32(0.50,3.49)$ \\
\hline Did not report seeing a physician this past year & $464(20.5)$ & $33(22.2)$ & $1.02(0.66,1.58)$ \\
\hline \multicolumn{4}{|l|}{$\mathrm{BMI}(\mathrm{SD})$} \\
\hline $18.5-24.9$ & $544(22.7)$ & $31(18.9)$ & $1.10(0.65,1.84)$ \\
\hline $25.0-29.9$ & $936(39.0)$ & $54(32.9)$ & ref. \\
\hline $30.0-34.9$ & $534(22.3)$ & $38(23.2)$ & $0.98(0.60,1.61)$ \\
\hline $35.0-39.9$ & $227(9.5)$ & $29(17.7)$ & $1.75(1.0,3.08)$ \\
\hline$\geq 40$ & $158(6.6)$ & $12(7.3)$ & $1.07(0.51,2.22)$ \\
\hline Hypertension & $1,969(80.4)$ & $144(86.8)$ & $1.11(0.66,1.87)$ \\
\hline Diabetes & $784(32.0)$ & $90(54.2)$ & $1.43(0.96,2.12)$ \\
\hline Coronary heart disease & $764(31.2)$ & $70(42.2)$ & $1.22(0.83,1.79)$ \\
\hline Stroke & $288(11.8)$ & $29(17.5)$ & $1.45(0.89,2.38)$ \\
\hline \multicolumn{4}{|l|}{ Urine albumin:creatinine ratio } \\
\hline$<30 \mathrm{mg} / \mathrm{g}$ & $1,640(67.0)$ & $60(36.1)$ & ref. \\
\hline $30-299.9 \mathrm{mg} / \mathrm{g}$ & $572(23.4)$ & $54(32.5)$ & $1.87(1.22,2.87)$ \\
\hline$\geq 300 \mathrm{mg} / \mathrm{g}$ & $237(9.7)$ & $52(31.3)$ & $2.14(1.26,3.61)$ \\
\hline \multicolumn{4}{|l|}{ eGFR } \\
\hline $45-59 \mathrm{ml} / \mathrm{min} / 1.73 \mathrm{~m}^{2}$ & $1,729(70.6)$ & $58(34.9)$ & ref. \\
\hline $30.0-44.9 \mathrm{ml} / \mathrm{min} / 1.73 \mathrm{~m}^{2}$ & $577(23.6)$ & $59(35.5)$ & $2.82(1.85,4.29)$ \\
\hline $15-29.9 \mathrm{ml} / \mathrm{min} / 1.73 \mathrm{~m}^{2}$ & $143(5.8)$ & $49(29.5)$ & $6.94(4.13,11.65)$ \\
\hline
\end{tabular}

* Adjusted for all covariates listed and region of residence; reference not listed for: education: > high school; income: $\geq 20,000 /$ year.

$\mathrm{n}=2,615$ for all rows except: health insurance $(\mathrm{n}=2,414)$; BMI $(\mathrm{n}=2,613)$; hypertension $(\mathrm{n}=2,563)$.

\section{Discussion}

Our main finding is that contrary to our hypothesis and regardless of the equation used to calculate eGFR, CKD awareness was associated with participation in only one healthy behavior thought to reduce the risk of adverse health outcomes among patients with CKD. CKD aware- ness appeared to be associated with higher odds of tobacco avoidance but not NSAIDs avoidance or participation in physical activity. Additionally, we found that an individual's awareness of having CKD was not associated with achievement of risk-reduction targets, including SBP control, among individuals with CKD with or without hypertension. Among individuals with CKD and dia- 
Table 2. ORs for participation in risk-reduction behaviors and achievement of risk-reduction targets if aware versus unaware of CKD

\begin{tabular}{llll}
\hline Behavioral outcome or risk reduction target & \multicolumn{2}{l}{ Adjusted OR (95\% CI) } & \\
\cline { 2 - 4 } & model 1 & model 2 & model 3 \\
\hline Tobacco avoidance & $1.51(0.88,2.61)$ & $1.48(0.85,2.59)$ & $1.82(1.02,3.24)$ \\
NSAID avoidance & $1.72(0.94,3.16)$ & $1.73(0.94,3.19)$ & $1.35(0.72,2.53)$ \\
Occasional physical activity (vs. none) & $0.97(0.67,1.39)$ & $1.10(0.74,1.54)$ & $1.21(0.83,1.78)$ \\
Frequent physical activity (vs. none) & $0.59(0.38,0.93)$ & $0.66(0.42,1.03)$ & $0.76(0.48,1.21)$ \\
SBP $<140$ mm Hg & $0.82(0.58,1.16)$ & $0.87(0.61,1.23)$ & $1.10(0.74,1.63)$ \\
SBP $<130$ mm Hg & $0.99(0.71,1.36)$ & $1.08(0.77,1.50)$ & $1.30(0.91,1.86)$ \\
ACEI/ARB use & $1.37(0.98,1.91)$ & $1.04(0.73,1.50)$ & $1.10(0.76,1.60)$ \\
\hline
\end{tabular}

Model 1: Adjusted for age, race, gender, marital status, income, education, and region. Model 2: Adjusted for model $1+$ comorbid conditions (high blood pressure, diabetes, stroke, coronary heart disease). Model 3: Adjusted for model $2+$ eGFR and urine albumin:creatinine ratio.

Table 3. ORs for achieving risk-reduction targets if aware versus unaware of CKD

\begin{tabular}{lccc}
\hline & Model 1 & Model 2 & Model 3 \\
\hline Among those with CKD and self-reported hypertension $(\mathrm{n}=2,102)$ & & \\
SBP $<140 \mathrm{~mm} \mathrm{Hg}$ & $0.82(0.57,1.18)$ & $0.83(0.57,1.20)$ & $1.06(0.71,1.57)$ \\
SBP $<130 \mathrm{~mm} \mathrm{Hg}$ & $0.99(0.79,1.39)$ & $1.00(0.71,1.43)$ & $1.27(0.87,1.85)$ \\
ACEI/ARB use & $1.31(0.90,1.92)$ & $1.13(0.76,1.67)$ & $1.27(0.84,1.91)$ \\
Among those with CKD and self-reported diabetes $(\mathrm{n}=933)$ & & \\
Fasting blood sugar $<130 \mathrm{mg} / \mathrm{dl}$ & $1.05(0.67,1.63)$ & $1.08(0.69,1.70)$ & $1.05(0.65,1.69)$ \\
ACEI/ARB use & $0.83(0.52,1.34)$ & $0.81(0.50,1.33)$ & $0.91(0.55,1.52)$ \\
\hline
\end{tabular}

Model 1: Adjusted for age, race, gender, marital status, education, income, region. Model 2: Adjusted for model $1+$ comorbid conditions (high blood pressure, diabetes, stroke, coronary heart disease). Model 3: Adjusted for model $2+$ eGFR and urine albumin:creatinine ratio.

betes, CKD awareness was not associated with higher odds of ACEI/ARB use or glycemic control. These latter results are consistent with data from the National Health and Nutrition Examination Survey (NHANES) that suggest that CKD awareness is not associated with receipt of guideline-concordant care [19]. This study adds to the literature by confirming the data from prior studies using a different question to ascertain CKD awareness: 'Has a doctor or other health professional ever told you that you had kidney disease?' versus 'Have you ever been told you have weak or failing kidneys (excluding kidney stones, bladder infections, or incontinence)?' It also extends knowledge further by focusing on the association between awareness of kidney disease and healthy behaviors, a potential mediator between awareness and improved health.
The lack of association between CKD awareness and healthy behaviors, with the possible exception of tobacco avoidance, is concerning. Our finding that CKD awareness is independent of chronic NSAID use, the only CKDspecific behavior that we examined, is consistent with results from previous studies that examined self-reported NSAID use among patients with CKD [20]. Among NHANES participants with eGFR $30-59 \mathrm{ml} / \mathrm{min} / 1.73 \mathrm{~m}^{2}$, use of prescription NSAIDS and those available over-thecounter was estimated to be 0.3 and $5.5 \%$, respectively. NSAIDs are often distributed with written information describing the risks associated with chronic NSAID use. Limited health literacy, which is quite prevalent among patients with CKD [11], limits the usefulness of such written pamphlets [21]. NSAID avoidance by patients with CKD may thus rely heavily on adequate verbal informa- 
tion transfer between health care providers and patients. We were unable to differentiate between over-the-counter and prescription NSAID use, but our results suggest that providers may fail to convey relevant information about CKD-specific preventive behaviors to patients when discussing CKD. This may be particularly true among generalists, who care for the majority of patients with CKD and do not often engage in conversations about CKD risk factors or CKD prevention [22]. The patientprovider interaction may thus be the most important unmeasured confounder in this study.

Interestingly, CKD awareness was associated with tobacco avoidance but was not associated with increased physical activity, two healthy behaviors that are not CKDspecific, but are emphasized for cardiovascular risk prevention. The discordant results may stem from unmeasured variables that lead to impaired physical functioning and disability among individuals with CKD [23]. While unable to discern from this study, it is possible that CKD awareness is associated with behaviors associated with improved cardiovascular risk profiles but not with CKDspecific preventative behaviors.

Results may also be explained by misclassification of CKD awareness. In this study, CKD awareness is ascertained by a question similar to one used in the Kidney Early Evaluation Program cohort study [24]: 'Has a doctor or other health professional ever told you that you had kidney disease?' Individual CKD awareness in NHANES is determined with a different question: 'Have you ever been told you have weak or failing kidneys (excluding kidney stones, bladder infections, or incontinence)?' While data from both sources suggest a similar low prevalence of CKD awareness [10], neither question has been validated among patients with and without CKD. It is plausible that an individual with albuminuria or decreased eGFR understands the extent of her kidney damage and the importance of participation in healthy behaviors to prevent further renal damage, without recognizing her kidney disease or failing kidneys. Similarly, the terminology in the aforementioned questions may not be consistent with terminology used by providers who describe CKD to patients. With on-going debates about the definition of CKD in the nephrology community [25], accurate ascertainment of CKD awareness may not be possible at this time.

More speculative explanations may also account for the lack of association between CKD awareness and participation in healthy behaviors. Perhaps individuals with CKD do not engage in healthy behaviors despite knowledge because of the silent nature of kidney disease until its progression to ESRD. This is consistent with data suggesting that individuals aware of hypertension, another 'silent disease', do not engage in healthy lifestyles within the first 2 years after diagnosis, with the exception of tobacco cessation [26]. Additionally, high prevalence of depression among patients with CKD is well established [27], but the extent to which CKD awareness is associated with denial, worry, or resignation has not been thoroughly explored.

This study has several limitations. Because of the crosssectional design of the study, causal inferences cannot be made. We also relied on single measurements for eGFR and albuminuria, possibly resulting in misclassification of individuals with CKD. While it was not possible to exclude individuals with acute kidney injury from this analysis, it is unlikely that many individuals had acute kidney injury rather than $\mathrm{CKD}$, given that individuals were recruited from home, not a hospital setting. Additionally, with the low percentage of participants aware of CKD, we may lack adequate power to detect statistically significant differences among patient groups. It is important to note that modeling the impact of CKD awareness on tobacco avoidance became statistically significant only after adjustment for albuminuria and eGFR, which, though unlikely, may represent overadjustment. Lastly, we did not have information about participant-provider relationships, participant health literacy, and provider interventions, such as prescribing ACEI/ARB or giving subjects information about healthy behaviors.

Our results suggest that the link between CKD awareness and healthy behaviors is not straightforward. While increasing CKD awareness among individuals with kidney disease should empirically reduce complications of $\mathrm{CKD}$, additional research is needed to determine how best to raise awareness of CKD and translate that awareness into improved health outcomes. Next steps could include identification of terminology used by providers and patients to describe kidney disease as well as barriers to patient adoption of CKD-healthy behaviors. Meanwhile, clinicians who discuss CKD with patients may need to include concrete information about behaviors thought to slow CKD progression and prevent its cardiovascular consequences. In turn, this may lead to healthier lifestyles and better health outcomes among patients with CKD. 


\section{Acknowledgements}

\section{The REGARDS Investigators}

The authors acknowledge the participating investigators and institutions of REGARDS for their valuable contributions: The University of Alabama at Birmingham (Study PI, Statistical and Data Coordinating Center, Survey Research Unit): V. Howard, PhD, L. Wagner, MA, V. Wadley, PhD, R. Go, PhD, M. Safford, MD, E. Temple, PhD, M. Stewart, MSPH, J.D. Rhodes, BSN; Wake Forest University (ECG Reading Center): R. Prineas, MD, PhD; University of Vermont (Central Laboratory): Rebekah Boyle; Alabama Neurological Institute (Stroke Validation Center, Medical Monitoring): C. Gomez, MD, S. Bowling, MD; University of Arkansas for Medical Sciences (Survey Methodology): L. Pulley, PhD; University of Cincinnati (Clinical Neuroepidemiology): B. Kissela, MD, D. Kleindorfer, MD; Examination Management Services, Incorporated (In-Person Visits): A. Graham; Medical University of South Carolina (Migration Analysis Center): D. Lackland, DrPH; Indiana University School of Medicine (Neuropsychology Center): F. Unverzagt, PhD; National Institute of Neurological Disorders and Stroke, National Institutes of Health (primary funding agency): C. Moy, PhD.

This research project is supported by a cooperative agreement U01 NS041588 from the National Institute of Neurological Disorders and Stroke, National Institutes of Health, Department of
Health and Human Services (George Howard, PI). The content is solely the responsibility of the authors and does not necessarily represent the official views of the National Institute of Neurological Disorders and Stroke or the National Institutes of Health. Representatives of the funding agency have been involved in the review of the manuscript but not directly involved in the collection, management, analysis or interpretation of the data.

D.S.T. was supported by KL2RR024130 from the National Center for Research Resources and K23DK09485. M.S. and P.M. are partially supported by NHLBI R01 HL080477. C.Y.H. was supported by K24DK92291. Additional funding was provided by an investigator-initiated grant from Amgen, Inc. to D.G.W. Amgen did not have any role in the design and conduct of the study, the collection, management, data analysis, or interpretation of the data. The manuscript was sent to Amgen for internal review prior to submission for publication.

\section{Disclosure Statement}

D.G.W. serves on the National Nephrology Advisory Board for Amgen, Inc. M.S. and P.M. receive financial support from Amgen, Inc. for investigator-initiated grants.

\section{References}

1 Levey AS, Stevens LA, Schmid CH, Zhang YL, Castro AF 3rd, Feldman HI, Kusek JW, Eggers P, Van Lente F, Greene T, Coresh J: A new equation to estimate glomerular filtration rate. Ann Intern Med 2009;150:604-612.

$\checkmark 2$ Tonelli M, Wiebe N, Culleton B, House A, Rabbat C, Fok M, McAlister F, Garg AX: Chronic kidney disease and mortality risk: a systematic review. J Am Soc Nephrol 2006;17: 2034-2047.

3 Sarnak MJ, Levey AS, Schoolwerth AC, Coresh J, Culleton B, Hamm LL, McCullough PA, Kasiske BL, Kelepouris E, Klag MJ, Parfrey P, Pfeffer M, Raij L, Spinosa DJ, Wilson PW: Kidney disease as a risk factor for development of cardiovascular disease: a statement from the American Heart Association councils on kidney in cardiovascular disease, high blood pressure research, clinical cardiology, and epidemiology and prevention. Hypertension 2003;42:1050-1065.

4 Regalado M, Yang S, Wesson DE: Cigarette smoking is associated with augmented progression of renal insufficiency in severe essential hypertension. Am J Kidney Dis 2000;35: 687-694.

5 Toyama K, Sugiyama S, Oka H, Sumida H, Ogawa $\mathrm{H}$ : Exercise therapy correlates with improving renal function through modifying lipid metabolism in patients with cardiovascular disease and chronic kidney disease. J Cardiol 2010;56:142-146.
6 CMS Manual System, Coverage of Kidney 12 Mason J, Khunti K, Stone M, Farooqi A, Carr Disease Patient Education Services: In: De- S: Educational interventions in kidney disease partment of Health and Human Services care: a systematic review of randomized trials. (DHHS) CfMMC (ed), 2009.

7 Devins GM, Mendelssohn DC, Barre PE, Binik YM: Predialysis psychoeducational intervention and coping styles influence time to dialysis in chronic kidney disease. Am J Kidney Dis 2003;42:693-703.

8 Manns BJ, Taub K, Vanderstraeten C, Jones $\mathrm{H}$, Mills C, Visser M, McLaughlin K: The impact of education on chronic kidney disease patients' plans to initiate dialysis with selfcare dialysis: a randomized trial. Kidney Int 2005;68:1777-1783.

9 Devins GM, Mendelssohn DC, Barre PE, Taub K, Binik YM: Predialysis psychoeducational intervention extends survival in CKD: a 20-year follow-up. Am J Kidney Dis 2005; 46:1088-1098.

10 Plantinga LC, Boulware LE, Coresh J, Stevens LA, Miller ER 3rd, Saran R, Messer KL, Levey AS, Powe NR: Patient awareness of chronic kidney disease: trends and predictors. Arch Intern Med 2008;168:2268-2275.

11 Wright JA, Wallston KA, Elasy TA, Ikizler TA, Cavanaugh KL: Development and results of a kidney disease knowledge survey given to patients with CKD. Am J Kidney Dis 2011;57: 387-395.
13 Kuo HW, Tsai SS, Tiao MM, Liu YC, Lee IM, Yang CY: Analgesic use and the risk for progression of chronic kidney disease. Pharmacoepidemiol Drug Saf 2010;19:745-751.

14 Jafar TH, Stark PC, Schmid CH, Landa M, Maschio G, de Jong PE, de Zeeuw D, Shahinfar S, Toto R, Levey AS: Progression of chronic kidney disease: the role of blood pressure control, proteinuria, and angiotensin-converting enzyme inhibition. A patient-level meta-analysis. Ann Intern Med 2003;139:244-252.

15 Howard VJ, Cushman M, Pulley L, Gomez CR, Go RC, Prineas RJ, Graham A, Moy CS, Howard G: The reasons for geographic and racial differences in stroke study: Objectives and design. Neuroepidemiology 2005;25:135-143.

16 National Kidney Foundation: Http://www. Kidney.Org/professionals/kdoqi/guidelines_ commentaries.Cfm\#guidelines.

17 Standards of Medical Care in Diabetes - 2006. Diabetes Care 2006;29(suppl 1):S4-S42.

18 Levey AS, Coresh J, Greene T, Stevens LA, Zhang YL, Hendriksen S, Kusek JW, Van Lente F: Using standardized serum creatinine values in the modification of diet in renal disease study equation for estimating glomerular filtration rate. Ann Intern Med 2006;145: 247-254. 
19 Tuot DS, Plantinga LC, Hsu CY, Powe NR: Is awareness of chronic kidney disease associated with evidence-based guideline-concordant outcomes? Am J Nephrol 2012;35:191197.

20 Plantinga L, Grubbs V, Sarkar U, Hsu CY, Hedgeman E, Robinson B, Saran R, Geiss L, Burrows NR, Eberhardt M, Powe N: Nonsteroidal anti-inflammatory drug use among persons with chronic kidney disease in the United States. Ann Fam Med 2011;9:423430.
21 Miller MJ, Schmitt MR, Allison JJ, Cobaugh DJ, Ray MN, Saag KG: The role of health literacy and written medicine information in nonsteroidal antiinflammatory drug risk awareness. Ann Pharmacother 2010;44:274-284.

22 Greer RC, Cooper LA, Crews DC, Powe NR, Boulware LE: Quality of patient-physician discussions about $\mathrm{CKD}$ in primary care: a cross-sectional study. Am J Kidney Dis 2011; 57:583-591.

23 Plantinga LC, Johansen K, Crews DC, Shahinian VB, Robinson BM, Saran R, Burrows NR, Williams DE, Powe NR: Association of CKD with disability in the United States. Am J Kidney Dis 2011;57:212-227.

-24 Whaley-Connell A, Sowers JR, McCullough PA, Roberts T, McFarlane SI, Chen SC, Li S, Wang C, Collins AJ, Bakris GL: Diabetes mellitus and CKD awareness: the Kidney Early Evaluation Program (KEEP) and National Health and Nutrition Examination Survey (NHANES). Am J Kidney Dis 2009;53:S11S21.
25 Parmar MS: Time to differentiate 'decreased kidney function' from 'kidney disease': towards improving the definition of chronic kidney disease. Int Urol Nephrol 2012;44: 493-497.

26 Neutel CI, Campbell NR: Changes in lifestyle after hypertension diagnosis in Canada. Can J Cardiol 2008;24:199-204.

27 Hedayati SS, Minhajuddin AT, Toto RD, Morris DW, Rush AJ: Prevalence of major depressive episode in CKD. Am J Kidney Dis 2009;54:424-432. 